\title{
FINDING THE LAND, PLANTING FIRST SEEDS; LEAD USER RESEARCH IN EARLY STAGE DESIGN FOR INTELLIGENT ECOSYSTEMS
}

\author{
V. Pannunzio ${ }^{\otimes}$, M. Kleinsmann, C. Duarte and D. Snelders \\ Delft University of Technology, The Netherlands \\ $\triangle$ v.pannunzio@tudelft.nl
}

\begin{abstract}
This contribution explores the potential of lead user research for early-stage designing for intelligent ecosystems through a literature review and a single case study concerning a lead user research initiative on blood pressure monitoring. The results suggest advantages of executing lead user research in early-stage designing for intelligent ecosystems from the points of view envisioning broad initial ecosystem boundaries, developing first intelligence components, and overcoming research challenges related to technical issues.
\end{abstract}

Keywords: systems engineering (SE), open innovation, healthcare design

\section{Introduction}

Designers who set out to develop complex digital ecosystems face a chicken-and-egg issue. On one hand, these kinds of propositions usually acquire their value by gaining a wide user base. On the other hand, it is problematic to attract a wide user base without offering tangible value. Hanseth and Lyytinen (2010) define this as the bootstrap problem of information infrastructures design, and examine the case study of the Internet itself to shed light on ways in which designers can deal with the issue.

They report how, unlike standard design processes, the development of what became later known as the Internet did not look like a problem solving activity; and quote Kahn, one of the initial developers of Internet protocols, reflecting:

'They (DoD) didn't have a problem. And that's why it's so hard for those kinds of things to actually get in motion. If you're saying, 'Can I imagine a problem that somebody might have at some unspecified point in the future?' Absolutely, that was what was driving it'.

Such reflections constitute a step further in our understanding of digital ecosystems design, but at the same time open puzzling questions. Where to find future problems? How to proceed with building germinal prototypes of what can only later evolve into a meaningful and functional proposition? Which 'seeds' to plant, and 'where' to plant them, to let a flourishing digital 'garden' grow?

In this contribution, we examine the method of Lead User research (von Hippel, 1986) as a way for designers to navigate the uncertainties posed by early-stage digital ecosystem design. Next, a brief review is offered on both ecosystem design and lead user research literature. 
Following, a single case study concerning a lead user research study in the context of smart blood pressure monitoring is presented. From it, key insights concerning the role of lead user research in early-stage design for intelligent ecosystems are illustrated and their relevance discussed. Finally, a three-phase model is proposed that integrates the insights offered in this contribution with a broader perspective on intelligent ecosystems design and management.

\section{Literature review}

\subsection{Intelligent ecosystems and design}

\subsubsection{Defining ecosystems and their intelligence}

The notion of 'ecosystem' in digital innovation literature is open to multiple interpretations and flavours. Among the main ones, we find the concepts of;

- Business ecosystems, intended as interconnected populations of organizations, be them 'small firms, large corporations, universities, research centres, public sector organizations and other parties which influence the system' (Peltoniemi and Vuori, 2004); and

- Digital or technological ecosystems, intended as 'solutions based on the composition of different software components and services that share a set of semantically defined data flows' (García-Peñalvo, 2018).

While these two characterizations describe different phenomena and arise from different disciplinary perspectives, they can meet in practice, since same entities can satisfy both sets of requirements (Figure 1). In the health and wellness domain, for instance, personal health apps such as Google Fit and Apple Health appear to be identifiable;

- As business ecosystems, since they constitute platforms which enable business interdependencies with and across digital products and services from other organizations; and

- As digital or technological ecosystems, since they are solutions which rely upon the interconnections with other software components and services, such as third party apps or connected devices.

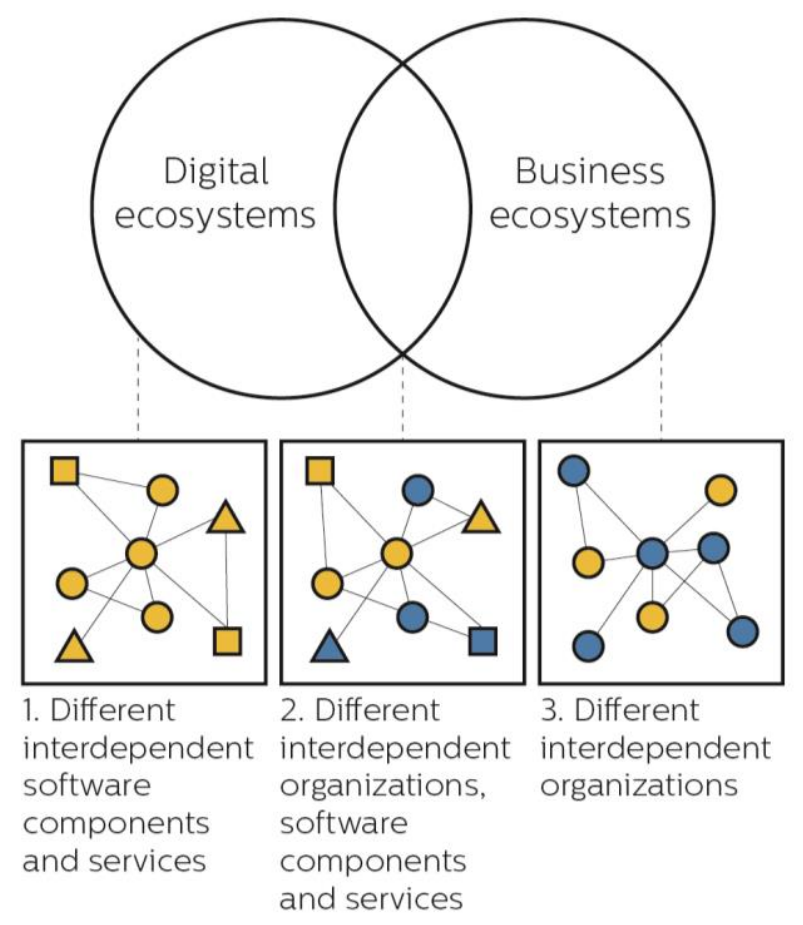

Figure 1. Digital ecosystems, business ecosystems, and their overlap 
Both these ecosystem characterizations - and their overlap - appear to be relevant from a design perspective. As noted by Gardien et al. (2014), designers wanting to work in digital ecosystems scenarios need to 'anticipate and design for the complexity, openness, and growth of these ecosystems'; while business ecosystems characterized by a variety of organizational players open new scenarios for design as a discipline, which 'has a key role to play in bringing these parties together'.

The design perspective is, also, one from which it makes sense to consider both these aspects of 'ecosystematicity' at the same time.

This is because design practice is concerned with and driven by user experiences, which are impacted upon both by the technical and by the business interdependencies of the designed propositions. For instance, we can imagine that the experience of using Google Fit or Apple Health would be considerably different if these were an expression of stand-alone software, or only interoperable with other apps from the same brand. Therefore, in this contribution, which aims at exploring design challenges and opportunities, we will adopt a broad definition of 'ecosystem', taking into consideration the digital characterization, the business one, and their overlap.

From a design perspective, it is also worth noting that in these kinds of ecosystems, as in many other digital domains, we can encounter the attribute of intelligence. Intelligence is, in fact, a feature achievable at an ecosystem level through the inclusion of AI components within the individual digital components of the ecosystem - or across their interconnections. In such conditions, ecosystems as a whole can acquire the capacity to evolve and adapt based on the inputs provided by their intelligent components, therefore achieving the status of intelligent ecosystem.

Incidentally, it must be observed that entities which can be recognized both as digital and as business ecosystems tend to constitute a fertile ground for intelligence. This is because, through their enabling of linkages between databases from different organizations, these entities can facilitate the aggregation of the large volumes of data usually necessary for the training of intelligent agents (Lee and Ha, 2018). Therefore, a focus will be maintained in this contribution on the aspect of intelligence within the ecosystems domain.

\subsubsection{Designing for intelligent ecosystems}

While intelligent ecosystems are relatively new in design literature, examples can be found of authors who explicitly addressed the theme. Hadzic et al. (2007), for instance, propose a bio-inspired method for the design of intelligent ecosystems, based on a five-step process which takes into account the design of every single digital component (or digital specie) in the ecosystem. The field of applicability of such a method appears, however, to be limited to situations in which the design of the ecosystems lies and is forecasted to lie in the hands of a single entity or organization.

Van Kollenburg and Bogers, on the other hand, propose an approach aimed at designing so to let intelligent ecosystems emerge, thus leaving space for further iterations and interpolations. They argue that 'the openness and complexity' associated with designing within the domain of intelligent ecosystems requires 'creative and generative approaches in which data can be used as a creative design material' (Van Kollenburg and Bogers, 2019). They propose a design research approach in which open-ended digital prototypes, situated in participants' everyday life, are used both to gain contextual, behavioural and experiential insights and to remotely conceptualize and deploy new design interventions. Through such continuous loop of in-situ iterations, first sprouts of intelligence can emerge, which the designers can then detail and expand.

\subsection{Lead user research as a candidate method for ecosystem design}

\subsubsection{Defining lead users research}

The 'lead user' construct was first introduced by von Hippel (1986) within a larger corpus of research on Open Innovation. Chesbrough (2006) defines Open Innovation as 'a paradigm that assumes that firms can and should use external ideas as well as internal ideas, and internal and external paths to market, as they look to advance their technology'. Lead users research is one of the several techniques contemplated in Open Innovation literature as a way to source external ideas; specifically, the ideas coming from the most advanced users. Importantly, these users are defined as 'advanced' not in reason 
of their knowledge and skills, but in the sense that their needs in terms of products or services exceeds what is available to them in the market (Eisenberg, 2011). Because of this unsatisfied need, lead users have a stronger-than-average motivation and probability to come up with new ideas and solutions to fulfil that need.

Empirical research confirms that the more users display lead user characteristics, the more likely it is that they will develop commercially attractive innovations (Franke et al., 2005).

The lead user research technique, initially developed by von Hippel et al. (2009), is in many ways similar to routine market or user research. Eisenberg (2011) defines four main differences between lead user research and other kinds of user research, clarifying that lead user research projects:

1. 'Focus on the needs of leading-edge users, not routine users;

2. Seek not only needs data but innovations - user-developed solutions to leading-edge needs from users;

3. Seek needs and solutions in adjacent markets and nonobvious, analogous markets, in addition to target markets;

4. Employ a cross-disciplinary team, bringing in perspectives from various parts of the organization.'

Lead users may not necessarily be identified as single individuals, but also as groups or companies (Eisenberg, 2011). Online or virtual communities, especially, have received attention in lead user research literature due to the opportunity of conducting participant recruiting through netnography techniques (Belz and Baumbach, 2010). Increasingly, lead user research is conducted through digital channels and with lead user communities who are 'naturally' aggregated online, through catalysts such as specialized websites, fora, chatrooms, and content platforms.

An important caveat in lead user research concerns the selection of the new product development stage at which to employ the technique. Gemser and Perks (2015) remark how lead user research may be more useful in the idea generation stage, to come up with the more radical ideas, while it may be more effective to involve 'traditional' users in ideas selecting and testing as they better represent the market. In the same way, Ebner et al. (2009) identify the main potential of conducting lead user research with virtual communities at the first stage of new product development (see Figure 2).

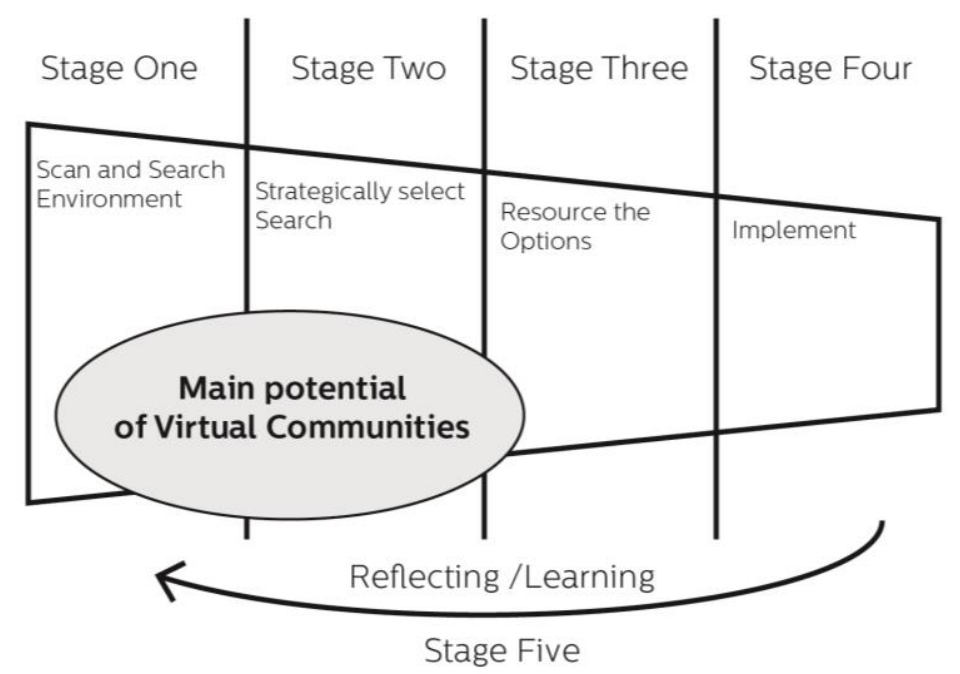

Figure 2. Process of innovation management and potential support of virtual communities (adapted from Ebner et al., 2009)

\subsubsection{Leading into intelligence}

The domain of intelligent ecosystem presents a few characteristics that can suggest potential advantages in employing lead user research techniques. These are; 
- Low technological and market maturity.

According to Tidd and Bessant (2005), the usefulness of lead user involvement is maximized in circumstances of low technological and market maturity, when it is necessary for the technological and market aspects to co-evolve until the first viable solutions emerge. Lead user research can then constitute a way to peek into the first indications of what the future could look like in a certain sector. Especially in their early stages, these circumstances apply to intelligent ecosystem propositions, which usually rely on advanced software and present the fast-growth patterns typical of unripe markets.

- Variety as a value.

Al-Zu'bi and Tsinopoulos (2012) highlight the positive impact of lead user research on the capacity of firms to increase the variety of their offerings. In an intelligent ecosystem scenario, offering variety is a crucial value driver, useful to consider both for innovating platform managers and complementors. For platform managers, lead user research may provide insights relevant to the management of the diversity of the platform modules, while for complementors lead user research may provide insights relevant to the discovery of areas of competitive advantage over fellow platform complementors.

- Quick-speed industry.

Dynamicity is identified as one of the key characteristics of the intelligent ecosystem domain. To innovate in such an environment, a forward-looking, trend-anticipating approach becomes a priority. Lead users, with their 'understanding of future needs' (Eisenberg, 2011), may therefore constitute an early source of knowledge for innovation. Moreover, Tsinopoulos and Al-Zu'bi (2012) report on positive effects of lead user involvement on new product development speed.

Finally, it is worth noting that lead user research is not new to the relatively young history of intelligent ecosystem design. An example can be found in the Philips Hue ecosystem, a smart home lighting solution which can interface with other domotics systems such as Google Home, supports connected bulbs from various brands, and even third-party apps. As reported in Hillbolling et al. (2017), the development of Hue has been inspired by the creativity and emergent behaviour of amateur tinkerers and coders who had 'great and fantastic ideas' about things that could be done with smart illumination.

Yet, few studies to date have focused explicitly on the role and potential of executing lead user research in order to support designers navigate the uncertainties posed by early-stage intelligent ecosystem design. This topic is thus explored in the following case study, which sets out to answer the research question: how can lead user research support early-stage designing for intelligent ecosystems?

\section{Case study: the Quantified Heart}

The Quantified Heart study is a lead user research activity focusing on emerging blood pressure selfmonitoring practices among lead users of self-tracking technology adopters. Participants were recruited among expert users of self-tracking devices and systems, including members of the Quantified Self community, an international community of users and makers of self-tracking tools who share an interest in "self-knowledge through numbers" (see Lupton, 2016).

The Quantified Self community was, in the context of this study, identified as an existing Virtual Community (Ebner et al., 2009).

The Virtual Community paradigm was embraced and embedded in the study setup through the organization of a remote research setting: participants were shipped the self-monitoring instrumentation and the main research activities were conducted through webinars, chatrooms, and individual video calls. The study included 14 participants from various continents, including the two researchers, who participated in the study in the first person. The setup included individual selfmonitoring of blood pressure and other data points deemed complementary or potentially interesting by the participants. During the active part of the study, which lasted for five months, experiences on the self-monitoring activities were routinely shared among the group, together with data visualization and interpretation tools, relevant knowledge sources, observations, and ideas. 
The research was performed according to the principles of the Helsinki Declaration and Nuremberg Code and was checked and reviewed by the human research ethics committee (HREC) of the Delft University of Technology. The participation in the research was voluntary, and the methods and data used were checked and approved by the HREC and the data steward of the Industrial Design Engineering faculty.

Raw data from the study, in the form of webinars and individual calls recordings, chatroom logs, and survey results, was analysed to distil relevant observations, needs, and ideas expressed by the participants. These, in turn, were compared with analogous material collected in a separate user research activity conducted on the same topic and through a comparable setup with eight 'routine' users. The two sets of results were compared and contrasted, until three main themes emerged. An overview of these findings is presented in the next section.

\section{Findings}

\subsection{Core vs peripheral needs}

The first difference in the sets of results from the two studies was individuated in the kind of user needs expressed by participants in relation to blood pressure monitoring. These appeared to be at the same time more diverse and more specialized in the lead user study, compared to the study conducted with routine users. Routine users appeared to be mostly interested in 'core', relatively predictable ecosystem functionalities, such as the possibility to share blood pressure data with their general practitioners. On the other hand, among lead users, several examples could be found of envisioned ecosystem functionalities that could hardly be predicted in advance by the design researchers. Examples of wishes articulated by lead users included, for instance, the desire to gain insights into the relationships between blood pressure fluctuations and personally-relevant metrics such as tremor intensity, medication intake, or menstrual cycle. All in all, routine users appeared to mostly express general, widely applicable ecosystem needs and wishes, while lead users expressed both general and 'niche', peripheral, specialized ones. This effectiveness of lead user research in surfacing 'peripheral' needs can be interpreted as instrumental to early-stage ecosystem design, in the measure in which it did help design researchers to appraise the full range of an ecosystem possibilities. This suggests that lead user research can be employed for defining the 'extreme' boundaries of the intelligent ecosystem market space, which can then be strategically segmented through routine user research. In early-stage designing for intelligent ecosystems, realizing the potential width of an ecosystem appears to be relevant for designers who want to mitigate later risks of ecosystem lack of variety and flexibility, over-specialization, and premature aging.

\subsection{Intelligence needs vs intelligence solutions}

A second difference observed between the two studies concerned the fact that while routine users mostly expressed needs, wishes, concerns, and experiences, lead users articulated all of the former in addition to ideas and solutions. Importantly, these ideas and solutions concerned intelligence aspects too. Several conversations among lead user participants were spent speculating on possible ways to develop intelligent functionalities. These regarded, for instance, the possibility to predict measurement errors based on past personal results, to provide personalized feedback on the effects of physical exercise on blood pressure through interpolation of blood pressure data with activity data from a person's smartphone or fitness tracker, or to program automatic blood pressure data 'cleaning' through machine learning algorithms comparing blood pressure data with variables from other devices, such as such as heart rate, date-time, or temperature.

This insight, which represents a confirmation of general lead user theory, presents specific implications in the domain of intelligent ecosystems design, in which the technicalities related to intelligent components might be unfamiliar to designers themselves.

Especially, this insight indicates that employing lead users in data-enabled design projects may facilitate the emergence of first intelligence components, playing a crucial role in informing the design of 'minimum viable' ecosystems. 


\subsection{High vs low tolerance for technical issues}

A final difference observed in the two groups concerned the attitude towards technical issues related to the data collection activities and the related self-monitoring devices. While the insurgence of such issue constituted in some cases a hard obstacle for conducting the research with routine users, lead users tended to be more tolerant towards these issues. In some cases, they even proactively found solutions design researchers were not aware of. An example in this sense concerns the Bluetooth pairing of the blood pressure monitors. Soon enough in the lead users study, it was discovered that participants based in other continents could not download the app used and suggested by the researchers; consequently, they could not retrieve blood pressure data from the monitoring devices. While the researchers were still looking for solutions, one of the participants found another app, compatible with the blood pressure device and downloadable irrespective of location. In this way, a fatal issue which would have severely compromised the viability of the study could be solved expeditiously. This highlights the convenience of conducting pilots with lead users before organizing data-enabled efforts with routine users as a way for design researchers to troubleshoot technical issues and be better prepared for unexpected occurrences.

\section{Discussion}

\subsection{Findings relevance and positioning}

The characterization of diverse, dynamic and self-evolving software platforms as 'ecosystems' builds on an analogy with the natural world. The 'ecosystem' metaphor builds on a set of analogies drawn between the main components of natural ecosystems (the organisms or biotic factors, the characteristics of their environment or abiotic factors, and the sets of relationships between organisms in the environment) and the main components of technological or software ecosystems (respectively, the stakeholders and software components, their business and digital environment, and the sets of relationships between components in the environment in the form of value exchanges or data flows).

Hillbolling et al. (2017) carry this natural ecosystem metaphor further, and compare the Hue light ecosystem to a garden populated by a thousand flowers (the third-party apps). In doing so, they build on the initial suggestion of Boudreau (2012) given to digital platform owners to attract an amount of complementors sufficient to 'let a thousand flowers bloom'.

The garden metaphor can be used to frame the findings of this contribution and connect them to a broader perspective on intelligent ecosystems design and management. The findings presented in section 4.1. can be seen as a way for designers in early-stage ecosystems design to 'find the land', or obtain an overview on the landscape of possibly relevant intelligent features. Similarly, the findings presented in section 4.2. can be seen as a way for designers to collect first 'seeds', or potential selfgrowing intelligence components that can be later enriched and evolved.

An overview of the relations between these insights and the previous contributions from Boudreau (2012) and Hillbolling et al. (2017) is offered in Figure 3.
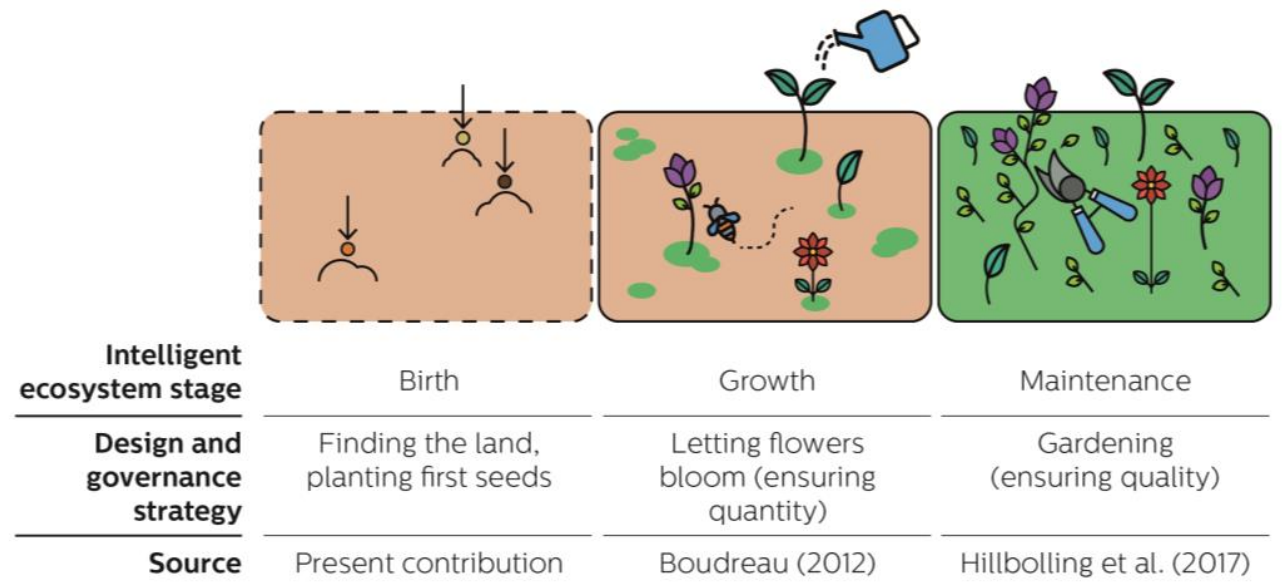

Figure 3. The intelligent ecosystem stage-based model (authors' own illustration) 


\subsection{Limitations and opportunities for further research}

The study presented in this contribution, and therefore the obtained results, presents definite limitations. The most important one concerns the fact that comparisons were drawn between two user studies of different size and duration. These limitations are highlighted as an opportunity for further research. Furthermore, to strengthen the reliability of the offered insight, further research in disparate ecosystem domains should be conducted comparing research activities following analogous setups.

Moreover, as already noted in section 2.2.2., it is relevant to remark that the presented advantages of employing lead user research are expected to be limited to cases of low technical and market maturity. While this currently applies to most products and services in the intelligent ecosystems domain, it is conceivable that in a near future the technical and market maturity of intelligent ecosystems will increase, thus diminishing the advantages of conducting lead user research in this domain.

Furthermore, it should be remarked that, both in lead user research and in data enabled design, special attention needs to be reserved to the ethics and transparency of research. Especially, existing Virtual Communities should not be exploited to generate data or ideas which will then be developed by separate, private institutions without their knowledge. Therefore, it is important to clarify research and design objectives upfront with all the involved participants, and to keep them informed about project developments even after the active phase of the study.

Relevant future research effort should further investigate on the complementarity of design research conducted with lead and routine users would and support the expansion and detailing of the stagebased model drafted in this contribution. Finally, as already observed in Schreieck et al. (2016), the role of data as a boundary resource in intelligent ecosystems is identified as a fruitful object for further design research.

\section{Conclusions}

The present contribution explored the potential of lead user research for early-stage designing for intelligent ecosystems. Through a literature review and a single case study concerning a lead user research initiative in the context of blood pressure monitoring, three main advantages for designers in executing lead user research in early-stage designing for intelligent ecosystems were identified. These are formulated as:

1. Support in envisioning broad initial ecosystem boundaries;

2. Support in developing first intelligence components;

3. Support in overcoming research challenges related to technical issues.

From these results, a stage-based model was proposed that integrates the provided insights with a broader perspective on intelligent ecosystem design and management. Finally, limitations were recognized and characterized as opportunities for further research.

\section{References}

Al-Zu'bi, Z.M.F. and Tsinopoulos, C. (2012), "Suppliers versus Lead Users: Examining Their Relative Impact on Product Variety", Journal of Product Innovation Management, Vol. 29 No. 4, pp. 667-680. http://dx.doi.org/10.1111/j.1540-5885.2012.00932.x

Belz, F.-M. and Baumbach, W. (2010), "Netnography as a Method of Lead User Identification", Creativity and Innovation Management, Vol. 19 No. 3, pp. 304-313. http://dx.doi.org/10.1111/j.1467-8691.2010.00571.x

Boudreau, K.J. (2012), "Let a Thousand Flowers Bloom? An Early Look at Large Numbers of Software App Developers and Patterns of Innovation", Organization Science, Vol. 23 No. 5, pp. 1409-1427. http://dx.doi.org/10.1287/orsc. 1110.0678

Chesbrough, H. (2006), “Open innovation: a new paradigm for understanding industrial innovation”, Open innovation: Researching a new paradigm, Vol. 400, pp. 0-19.

Ebner, W., Leimeister, J.M. and Krcmar, H. (2009), "Community engineering for innovations: the ideas competition as a method to nurture a virtual community for innovations", $R \& D$ Management, Vol. 39 No. 4 , pp. 342-356. http://dx.doi.org/10.1111/j.1467-9310.2009.00564.x

Eisenberg, I. (2011), "Lead-User Research for Breakthrough Innovation”, Research-Technology Management, Vol. 54 No. 1, pp. 50-58. http://dx.doi.org/10.5437/08953608x540150 
Franke, N., von Hippel, E.A. and Schreier, M. (2005), "Finding Commercially Attractive User Innovations: A Test of Lead User Theory", SSRN Electronic Journal. http://dx.doi.org/10.2139/ssrn.721182

García-Peñalvo, F.J. (2018), "Global Implications of Emerging Technology Trends", Advances in IT Standards and Standardization Research. http://dx.doi.org/10.4018/978-1-5225-4944-4

Gardien, P., Deckers, E. and Christiaansen, G. (2014), "Innovating Innovation: Enabling Meaningful Experience in Ecosystems", Design Management Journal, Vol. 9 No. 1, pp. 36-46.

Gemser, G. and Perks, H. (2015), "Co-Creation with Customers: An Evolving Innovation Research Field", Journal of Product Innovation Management, Vol. 32 No. 5, pp. 660-665. http://dx.doi.org/10.1111/jpim. 12279

Hadzic, M., Chang, E. and Dillon, T. (2007), "Methodology framework for the design of digital ecosystems", 2007 IEEE International Conference on Systems, Man and Cybernetics. http://dx.doi.org/10.1109/icsmc. 2007.4414235

Hanseth, O. and Lyytinen, K. (2010), "Design theory for dynamic complexity in information infrastructures: the case of building internet", Journal of information technology, Vol. 25 No. 1, pp. 1-19. https://doi.org/ $10.1057 /$ jit.2009.19

Hilbolling, S. et al. (2017). "Gardening a thousand flowers: Ensuring complement quality over time in the Philips Hue ecosystem”, In Academy of Management Proceedings (Vol. 2017 No. 1, p. 11805). Briarcliff Manor, NY 10510: Academy of Management. http://dx.doi.org/10.5465/ambpp.2017.11805abstract

Lee, K. and Ha, N. (2018, January), “AI platform to accelerate API economy and ecosystem", In 2018 International Conference on Information Networking (ICOIN), IEEE, pp. 848-852. https://doi.org/10.1109/ icoin.2018.8343242

Lupton, D. (2016), The quantified self: a sociology of self-tracking. John Wiley \& Sons.

Peltoniemi, M. and Vuori, E. (2004), "Business ecosystem as the new approach to complex adaptive business environments", Proceedings of eBusiness research forum, Vol. 2 No. 22, pp. 267-281.

Schreieck, M., Wiesche, M. and Krcmar, H. (2016), "Design and governance of platform ecosystems-key concepts and issues for future research".

Tidd, J. and Bessant, J.R. (2005), Managing innovation: integrating technological, market, and organizational change, John Wiley, Chichester.

Tsinopoulos, C. and Al-Zu'bi, Z. (2012), "Clockspeed effectiveness of lead users and product experts", International Journal of Operations \& Production Management, Vol. 32 No. 9, pp. 1097-1118. http://dx. doi.org/10.1108/01443571211265710

van Kollenburg, J. and Bogers, S.J.A. (2019), Data-enabled design: a situated design approach that uses data as creative material when designing for intelligent ecosystems [PhD thesis], Eindhoven University of Technology.

Von Hippel, E. (1986), "Lead Users: A Source of Novel Product Concepts”, Management Science, Vol. 32 No. 7, pp. 791-805, Available at: http://dx.doi.org/10.1287/mnsc.32.7.791.

Von Hippel, E., Churchill, J. and Sonnack, M. (2009), "Lead User Project Handbook: A practical guide for lead user project teams". 
